\title{
Aim and scope of the fellows forum
}

\author{
Susan S. Smyth
}

Published online: 3 January 2012

(C) Springer Science+Business Media, LLC 2012

Welcome to the first edition of the Fellows Forum of the Journal of Thrombosis and Thrombolysis. The Forum is a new, recurring feature that will appear on a regular basis in each volume of the journal. Its purpose is to recognize scholarly activities in clinical trainees participating in the continuum of cardiovascular science from bench to bedside and to encourage the next generation of clinician-scientists and investigators in the fields of cardiology, hematology, vascular medicine, and related disciplines.

The Forum is open to individuals engaged in a residency program, as well as those who are in fellowship training positions. The Forum will publish meritorious original work devoted to fundamental science in the areas of cardiovascular disease, preclinical and clinical research, global health, and epidemiology. In some instances, highly unique case reports, or ones that include multimedia and/or video, will also be presented. In addition to being a prominent and distinguished channel for trainees to publish their original research or reports, the Fellows Forum also provides residents and fellows the opportunity to engage in the peerreview and editorial process, often for the first time. The Forum plans to add an online platform, such as a social media page, which would serve as a medium where fellows and residents may submit work to be featured and discussed amongst peers.

We invite you to read and enjoy the innovative work presented here in the Forum, knowing that you may be reading the very first article from an emerging leader in our field.

For more information or to submit a manuscript, contact lisa.noble@springer.com and/or go to http://www.springer. com/medicine/cardiology/journal/11239.
S. S. Smyth $(\bowtie)$

Division of Cardiovascular Medicine, Lexington VA Medical Center and Gill Heart Institute, Lexington, KY 40356, USA

e-mail: ssmyt2@email.uky.edu 